LAPTH-1075/04

IFT-2004-28

hep-ph/0410248

\title{
Large 2HDM(II) one-loop corrections in leptonic tau decays
}

\author{
Maria Krawczyk ${ }^{1}$ and David Temes ${ }^{2}$
}

1. Institute of Theoretical Physics, University of Warsaw, ul. Hoża 69

Warsaw, 00-681, Poland

2. Laboratoire de Physique Théorique LAPTH

Ch. de Bellevue, B.P 110, F-74941, Annecy-le-Vieux, Cedex, France. ${ }^{3}$

\begin{abstract}
The one-loop contributions to the branching ratios for leptonic $\tau$ decays are calculated in the $\mathrm{CP}$ conserving $2 \mathrm{HDM}(\mathrm{II})$. We found that these one-loop contributions, involving both neutral and charged Higgs bosons, dominate over the tree-level $H^{ \pm}$exchange, the latter one being totally negligible for the decay into $e$. The analysis is focused on large $\tan \beta$ enhanced contributions to the considered branching ratios. We derive a simple analytical expression for the one-loop contribution which holds in this case. We show that the leptonic branching ratios of $\tau$ are complementary to the Higgsstrahlung processes for $h(H)$ and have a large potential on constraining parameters of the model. In this work we provide upper limits on Yukawa couplings for both light $h$ and light $A$ scenarios and we derive new lower limit on mass of $M_{H^{ \pm}}$as a function of $\tan \beta$, which differs significantly from what was considered as standard constraint based on the tree-level $H^{ \pm}$exchange only. Interestingly we obtain also an upper limit on $M_{H^{ \pm}}$. For a SMlike $h$ scenario, with heavy and degenerate additional Higgs bosons, one-loop corrections disappear.
\end{abstract}

\footnotetext{
${ }^{3}$ UMR 5108 du CNRS, associée à l'Université de Savoie
} 


\section{Introduction}

The mechanism of electroweak symmetry breaking is the most important ingredient in the description of elementary particle physics. The Standard Model (SM) incorporates the Higgs mechanism that breaks the electroweak symmetry spontaneously through a neutral scalar field with non-zero vacuum expectation value. In the minimal version of this mechanism one scalar $S U(2)_{L}$ doublet is required, providing one physical particle: the Higgs boson. The search of this particle is one of the main aims of high energy physics and current searches at LEP exclude SM Higgs bosons with masses below $114.1 \mathrm{GeV}$ at $95 \%$ C.L. [1]. In this context, valuable information about the Higgs mass will come from analysis of precise measurements of electroweak observables. The result of these indirect searches gives an upper bound on the Higgs mass $M_{H_{S M}}<219 \mathrm{GeV}$ at $95 \%$ C.L. [1], that is of great importance for future searches.

Models with Two Higgs Doublets (2HDM) are the minimal extensions of the SM Higgs sector describing all high energy experimental data and providing new and rich phenomenology. These models can also be interpreted as effective theories describing low-energy physics in models with beyond the SM physics at higher scale. This maybe the case of the Minimal Supersymmetric Standard Model (MSSM) with heavy supersymmetric particles. A CP-conserving 2HDM contains 5 physical Higgs bosons, two neutral scalars, $h$ and $H$, one pseudoscalar $A$, and two charged Higgs bosons, $H^{ \pm}$(see e.g. [2]). The LEP direct searches for these Higgs bosons are more complicated than in the SM due to the number of free parameters involved and, in particular, the existence in 2HDM of one very light Higgs boson can not be excluded [1]. On the other hand, LEP data excludes, for example, neutral Higgs bosons with masses below $40 \mathrm{GeV}$ in the regime of large $\tan \beta$, equal 60 or larger [3].

In this context, indirect searches for $2 \mathrm{HDM}$ effects in electroweak observables provide important information about the masses and mixing angles in the Higgs sector. For example, concerning the charged Higgs, a lower bound $M_{H^{ \pm}}>490 \mathrm{GeV}$ can be set using indirect effects in $b \rightarrow s \gamma$ [4], to be compared with $M_{H^{ \pm}}>75.5 \mathrm{GeV}$ coming from the direct LEP searches [5]. In order to explore the whole parameter space, global fits using different electroweak observables $\rho, R_{b}$ and $b \rightarrow s \gamma[6,7]$ (and also $(g-2)_{\mu}$ in [7]), have been made, constraining large regions of the parameter space and therefore giving valuable information for future searches.

In this work, a complete study of one-loop 2HDM effects in the leptonic $\tau$ decays is performed for large $\tan \beta$ and arbitrary Higgs spectrum, extending the results from [8] and [9].

It will be seen that this radiative effects in the branching ratios for $\tau \rightarrow$ $e \bar{\nu}_{e} \nu_{\tau}$ and $\tau \rightarrow \mu \bar{\nu}_{\mu} \nu_{\tau}$ are larger that the 2HDM tree-level effects in the relevant regions of parameter space and experimental data will be used to derive new constraints for Higgs masses and mixing angles.

The paper is organised as follows. The Sect. 2 contains a short description 
of the 2HDM properties and of results of the experimental searches on Higgs bosons. In Sect. 3 the leptonic $\tau$ decay data are compared with the SM prediction and the $95 \% \mathrm{CL}$ bounds for $2 \mathrm{HDM}$ contributions to the branching ratios are derived. In Sect. 4 the $2 \mathrm{HDM}$ contributions are parameterised. The one-loop 2HDM effects are computed in Sect. 5 while their numerical analysis is performed in Sect. 6. Finally, in Sect. 7 we derived the constrains on the 2HDM parameters coming from leptonic $\tau$ decay data analysis and our conclusions are summarised in Sect. 8.

\section{CP conserving 2HDM Model II}

\subsection{General properties}

The Two-Higgs-Doublet Model is the simplest extension of the Standard Model with one extra scalar doublet. It contains three neutral and two charged Higgs bosons. Here we consider a simple CP conserving version with a soft $Z_{2}$-violation, assuming the Yukawa interactions according to the Model II, 2HDM(II), as in MSSM. In this model one of the Higgs scalar doublet couples to the up-components of isodoublets while the second one to the down-components. In this case there are 7 parameters describing the Higgs Lagrangian: four masses for $h, H, A$ and $H^{ \pm}$, two mixing angles $\alpha$ and $\beta$ (used in form $\sin (\beta-\alpha)$ and $\tan \beta=v_{2} / v_{1}$ ), and the $\nu$-parameter, related to the soft- $Z_{2}$ violating mass term in the Lagrangian $\left(\nu=\Re m_{12}^{2} / 2 v_{1} v_{2}\right)$. This $\nu$-parameter describes the Higgs selfcouplings if they are expressed in terms of masses. We stress that none of these selfcouplings are involved in this analysis directly. However our results are sensitive indirectly to the $\nu$ parameter as this parameter governs the decoupling properties of the model.

There is the attractive possibility of having a neutral Higgs boson $h$ similar to the SM one, and all other Higgs bosons much heavier. This scenario can be realised in two ways, depending on the value of $\nu$-parameter. For large $\nu$ the additional Higgs boson masses can be very large and almost degenerate, since all of such masses arise from one large parameter $-\nu$. It is well known that in such case there is decoupling of these heavy bosons from known particles, i.e. effects of these additional Higgs bosons disappear if their masses tend to infinity, eg. in the $\gamma \gamma h$ coupling. At small $\nu$ the large masses of such additional Higgs bosons arise from large quartic selfcouplings $(\lambda)$ in the Lagrangian. Since these couplings are bounded from above by the unitarity constraints, so are the heavy Higgs-boson masses. According to these bounds heavy Higgs bosons have to be, typically, lighter than $600 \mathrm{GeV}$ [10]. Therefore, in this scenario the additional Higgs bosons can be heavy enough to avoid direct observation even at next generation of colliders, although some relevant effects can appear in the interaction of the lightest Higgs boson (non-decoupling) [11-14].

Another interesting scenarios that will be intensively studied in this work

are the ones with mass of $h$ or $A$ below the SM Higgs boson mass limit, 
$114 \mathrm{GeV}$. In particular, the light $A$ scenario is especially relevant for the description of $(g-2)_{\mu}$ data [15-17]. These scenarios are possible without conflict with the existing data within a $2 \mathrm{HDM}(\mathrm{II})$, since this model allows for low production rates for very light Higgs particles, as will be discussed below.

The 2HDM (II) model is characterised by the couplings of Higgs bosons to the fermions and to the EW gauge bosons. Their ratios to the corresponding couplings in the SM, $\chi_{j}^{i}=g_{j}^{i} / g_{j}^{S M}$, are presented in Table 1 . Note that for couplings to the EW gauge bosons $V$,

$$
\left(\chi_{V}^{h}\right)^{2}+\left(\chi_{V}^{H}\right)^{2}+\left(\chi_{V}^{A}\right)^{2}=1
$$

and similarly for the couplings to fermions [11]. Note also, that for each neutral Higgs boson (i) we have

$$
\left(\chi_{u}^{i}+\chi_{d}^{i}\right) \chi_{V}^{i}=1+\chi_{u}^{i} \chi_{d}^{i}
$$

Table 1: Relative couplings, $\chi_{j}^{i}=g_{j}^{i} / g_{j}^{S M}$ in $2 H D M(I I)$

\begin{tabular}{|l|c|c|c|}
\hline & $h$ & $H$ & $A$ \\
\hline$\chi_{V}$ & $\sin (\beta-\alpha)$ & $\cos (\beta-\alpha)$ & 0 \\
$\chi_{u}$ & $\chi_{V}^{h}+\cot \beta \chi_{V}^{H}$ & $\chi_{V}^{H}-\cot \beta \chi_{V}^{h}$ & $-i \cot \beta$ \\
$\chi_{d}$ & $\chi_{V}^{h}-\tan \beta \chi_{V}^{H}$ & $\chi_{V}^{H}+\tan \beta \chi_{V}^{h}$ & $-i \tan \beta$ \\
\hline$\chi_{W^{-} H^{+}}$ & $\cos (\beta-\alpha)$ & $\sin (\beta-\alpha)$ & 0 \\
\hline
\end{tabular}

Note, that for large $\tan \beta$ the couplings to the charged leptons (equal to the couplings to the down-type quarks $\chi_{d}$ ), relevant for our analysis, are enhanced.

In the last row of Table 1] the $W^{ \pm} H^{\mp} \phi^{o}$ couplings, with $\phi^{o}=h, H, A$, being of interest of this work, are presented. Here the ratios of such couplings to the SM Higgs boson coupling to gauge boson, $\chi_{W^{-} H^{+}}^{i}=g_{W^{ \pm} H^{\mp}}^{i} / g_{W}^{S M}$, are shown.

It is important to notice the complementarity between the $\chi_{V}^{i}$ on one hand and $\chi_{W^{-} H^{+}}^{i}\left(\right.$ and $\chi_{d}^{i}$ at large $\left.\tan \beta\right)$ on the other.

\subsection{Experimental constraints on $2 \mathrm{HDM}$}

The most important constraints on the $2 \mathrm{HDM}(\mathrm{II})$ parameter space come from LEP direct searches for Higgs bosons. Concerning light neutral Higgs bosons production, there are three main processes within the energy range covered by LEP, namely, the Higgsstrahlung, $e^{+} e^{-} \rightarrow Z^{*} \rightarrow Z h$, the associated

production, $e^{+} e^{-} \rightarrow Z^{*} \rightarrow h A$, and the Yukawa processes, $e^{+} e^{-} \rightarrow f \bar{f} \rightarrow$ 
$f \bar{f} h(A)$. The two first processes are highly complementary, due to their dependence on $(\beta-\alpha)$,

$$
\begin{aligned}
\sigma\left(e^{+} e^{-} \rightarrow Z^{*} \rightarrow Z h\right) & =\sin ^{2}(\beta-\alpha) \sigma_{S M}\left(e^{+} e^{-} \rightarrow Z^{*} \rightarrow Z H_{S M}\right) \\
\sigma\left(e^{+} e^{-} \rightarrow Z^{*} \rightarrow h A\right) & =\cos ^{2}(\beta-\alpha) \sigma_{S M}\left(e^{+} e^{-} \rightarrow Z^{*} \rightarrow Z H_{S M}\right) \bar{\lambda} \\
\sigma\left(e^{+} e^{-} \rightarrow Z^{*} \rightarrow f \bar{f} h\right) & =\left(\chi_{d}^{h}\right)^{2} \sigma_{S M}\left(e^{+} e^{-} \rightarrow Z^{*} \rightarrow f \bar{f} H_{S M}\right) \\
\sigma\left(e^{+} e^{-} \rightarrow Z^{*} \rightarrow f \bar{f} A\right) & =\left(\chi_{d}^{A}\right)^{2} \sigma_{S M}\left(e^{+} e^{-} \rightarrow Z^{*} \rightarrow f \bar{f} H_{S M}\right)
\end{aligned}
$$

where $\bar{\lambda}=\lambda_{A h}^{3 / 2} /\left[\lambda_{Z h}^{1 / 2}\left(12 M_{Z}^{2} / s+\lambda_{Z h}\right)\right]$, with $\lambda_{i j}=\left(1-m_{i}^{2} / s+m_{j}^{2}\right)^{2}-$ $4 m_{i}^{2} m_{j}^{2} / s^{2}$, being the two-particle phase-space factor.

The search for a light $h$ through the Higgsstrahlung process, under an assumption that the light Higgs boson decays into hadronic states, has been performed in [18]. The results of this analysis set an upper limit on the product of the cross section and the corresponding branching ratio. It can be translated into an upper limit on $\sin ^{2}(\beta-\alpha)$ as a function of $M_{h}$, shown in Fig. 1 (left) [18]. Therefore, the results of this analysis are compatible with a light $h$ scenario (with mass below $114 \mathrm{GeV}$ ) if $\sin ^{2}(\beta-\alpha)$ is small enough.

Also upper limits on the cross section of the associated $h A$ production process have been derived assuming $100 \%$ decays into hadrons [19]. These results can be translated into forbidden regions in the $2 \mathrm{HDM}(\mathrm{II})$ parameter space. In particular, these results highly constrain a scenario with both $h$ and $A$ light (the light $A \& h$ scenario). In Fig. 1 the excluded $\left(M_{h}, M_{A}\right)$ regions have been plotted [19]. A particular point is excluded in Fig. 11 (right) if it is excluded for $0.4 \leq \tan \beta \leq 40$ (darker grey region), $0.4 \leq \tan \beta \leq 1$ (lighter grey region), and $1 \leq \tan \beta \leq 40$ (hatched region) for all values of $\alpha= \pm \pi / 2, \pm \pi / 4,0$. It is noticeable that a scenario with light $h(A)$ is not excluded if $M_{A}\left(M_{h}\right)$ is large enough. In particular, if $\sin ^{2}(\beta-\alpha)=0$, LEP measurements are sensitive to this associated production if $M_{h}+M_{A} \leq$ $130-140 \mathrm{GeV}$.

Finally, the search for a light Higgs boson has been performed through the analysis of Yukawa processes assuming that Higgs boson decays into $\tau$, if $2 m_{\tau}<M_{h}, M_{A}<2 m_{b}$, or into $b$-quarks, if $M_{h}, M_{A}>2 m_{b}$ [3]. One of the results of this analysis is that $M_{h, A} \leq 40 \mathrm{GeV}$ are excluded for high $\tan \beta$ $(\tan \beta \geq 60)$. We will discuss existing constraints together with new ones coming from our analysis in Sect.7.

Concerning the charged Higgs boson, direct searches at LEP through the process $e^{+} e^{-} \rightarrow H^{+} H^{-}$have been performed assuming $B R\left(H^{-} \rightarrow q \bar{q}\right)+$ $B R\left(H^{-} \rightarrow \tau \nu_{\tau}\right)=1$. The lower bound $M_{H^{ \pm}} \geq 75.5 \mathrm{GeV}$ at $95 \% \mathrm{CL}$ [5] was obtained. The Tevatron data set limits on mass of the charged Higgs boson as a function on $\tan \beta$, they are presented together with LEP results on Fig. 2 ,

Much stronger constraints on $M_{H^{ \pm}}$come from the indirect charged Higgs boson effects in $b \rightarrow s \gamma$ processes, if interpreted in $2 \mathrm{HDM}(\mathrm{II})$. This leads to a lower mass limit of $490 \mathrm{GeV}$ at $95 \%$ for $\tan \beta>2[4]^{4}$.

\footnotetext{
${ }^{4}$ Recent analysis on $B \rightarrow X_{s} \gamma$ predicts larger theoretical errors in the SM prediction
} 

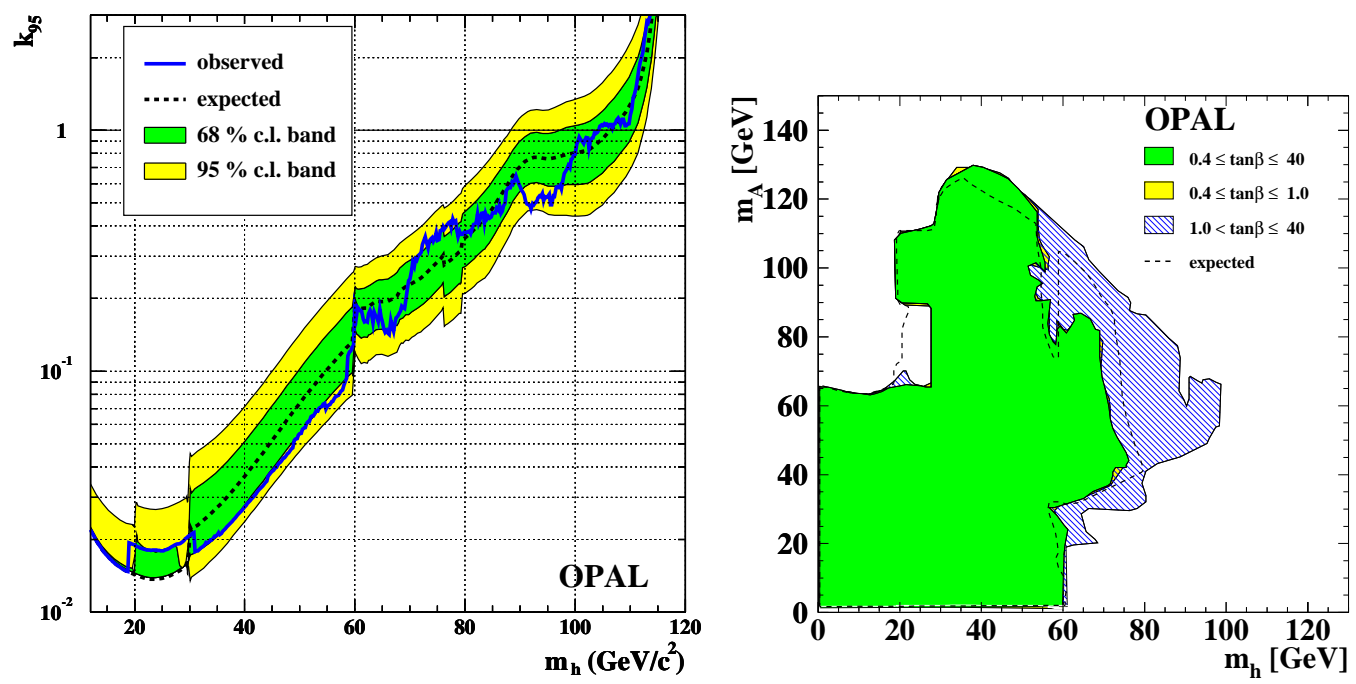

Figure 1: Left: Upper limit on $\sin ^{2}(\beta-\alpha)$ as a function of $M_{h}$ [18]; Right: Excluded $\left(M_{h}, M_{A}\right)$ regions in the different ranges of $\tan \beta$ for $\alpha=$ $\pm \pi / 2, \pm \pi / 4,0$ by OPAL [19].

In this context, important information on the available $2 \mathrm{HDM}(\mathrm{II})$ parameter space is coming indirectly from the low energy precise measurements. In particular, from the Upsilon decay into $h(A) \gamma$ and $g-2$ data, see eg. [17]. Also global fits have been performed, combining the results coming from the different electroweak observables $\rho, R_{b}$ and $b \rightarrow s \gamma[6,7]$ (and also $(g-2)_{\mu}$ in [7]), constraining large regions of the parameter space. Here, indirect constraints of $2 \mathrm{HDM}(\mathrm{II})$ will be obtained using leptonic $\tau$ decays data. The obtained results will be compared with direct search analysis coming from LEP and some low energy experiments. The implementation of leptonic $\tau$ decay data in global fits will be performed elsewhere.

\section{Leptonic $\tau$ decays: data versus SM predic- tions}

We consider the partial decay widths and branching ratios for the two leptonic decay channels of the $\tau$-lepton, namely

$$
\tau \rightarrow e \bar{\nu}_{e} \nu_{\tau} \text { and } \tau \rightarrow \mu \bar{\nu}_{\mu} \nu_{\tau} .
$$

We will denote the corresponding quantities using superscript $l, l=e$ and $\mu$, for example for the branching ratio we use $B r^{l}=B r\left(\tau \rightarrow l \bar{\nu}_{l} \nu_{\tau}\right)$.

The '04 world averaged data for the leptonic $\tau$ decay modes and $\tau$ lifetime and therefore a more conservative lower bound $M_{H^{ \pm}} \geq 200 \mathrm{GeV}[20]$. 


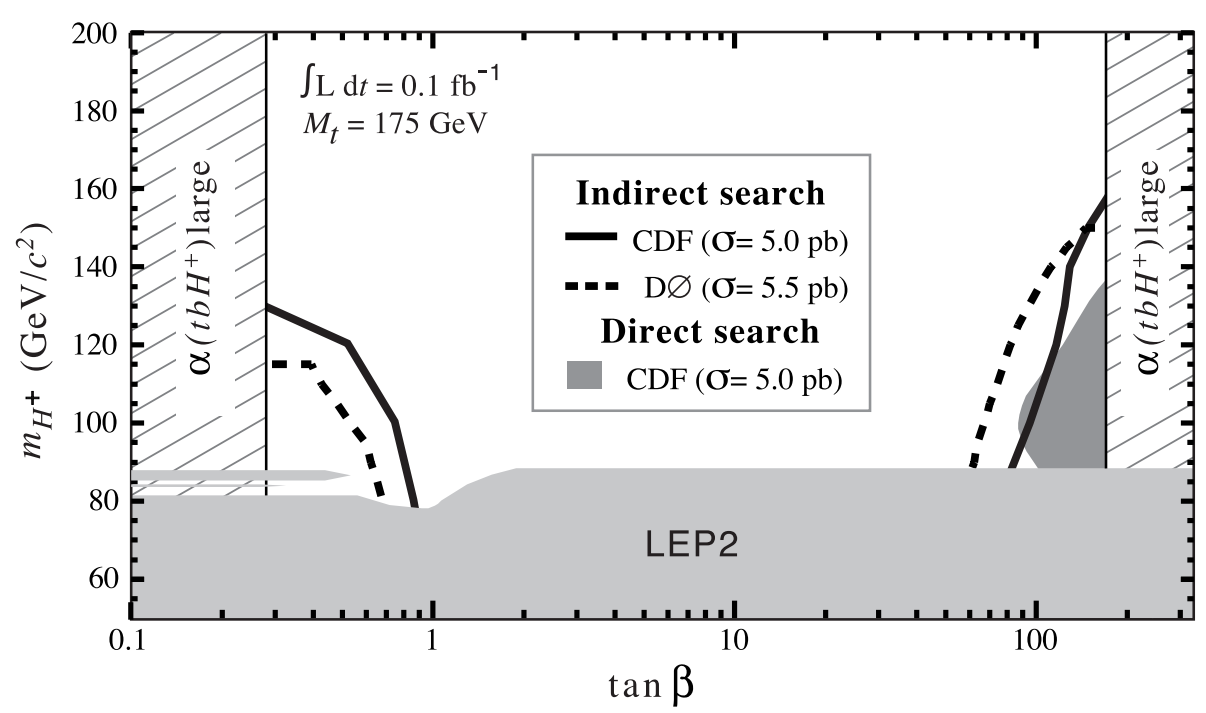

Figure 2: Constraints on charged Higgs boson mass [1].

are [1]

$$
\begin{gathered}
\left.B r^{e}\right|_{\exp }=(17.84 \pm 0.06) \%,\left.\quad B r^{\mu}\right|_{\exp }=(17.37 \pm 0.06) \% \\
\tau_{\tau}=(290.6 \pm 1.1) \times 10^{-15} s .
\end{gathered}
$$

Note that the relative errors of the above measured quantities are of the $0.34-0.38 \%$, the biggest being for the lifetime.

The SM prediction for these branching ratios can be defined as the ratios of the SM predicted decay widths to the total width as measured in the lifetime experiments, namely $\left.B r^{l}\right|_{S M}=\left.\Gamma^{l}\right|_{S M} / \Gamma_{\text {exp }}^{\text {tot }}=\left.\Gamma^{l}\right|_{S M} \tau_{\tau}$. Therefore, one can parameterise a possible beyond the SM contribution by a quantity $\Delta^{l}$, defined as

$$
B r^{l}=\left.B r^{l}\right|_{S M}\left(1+\Delta^{l}\right) .
$$

In the lowest order of SM the leptonic decay width of the $\tau$ is due to the tree level $W$ exchange, see Fig. 3 (left). Including the $\mathrm{W}$-propagator effect and QED radiative corrections, the following results for the branching ratios in the SM are obtained (see also Sect. 4):

$$
\left.B r^{e}\right|_{S M}=(17.80 \pm 0.07) \%,\left.B r^{\mu}\right|_{S M}=(17.32 \pm 0.07) \% .
$$

Together with the experimental data this leads to the following estimations for the possible beyond SM contributions to the considered branching ratios,

$$
\Delta^{e}=(0.20 \pm 0.51) \%, \quad \Delta^{\mu}=(0.26 \pm 0.52) \% .
$$

Using them we derive the $95 \%$ C.L. bounds on $\Delta^{l}$, for the electron and muon decay mode, respectively:

$$
\left(-0.80 \leq \Delta^{e} \leq 1.21\right) \%, \quad\left(-0.76 \leq \Delta^{\mu} \leq 1.27\right) \% .
$$

One can see that the negative contributions are constrained more strongly that the positive ones. 


\section{Leptonic $\tau$ decays in 2HDM}

In the SM the leptonic $\tau$ decay, $\tau \rightarrow l \bar{\nu}_{l} \nu_{\tau}$, proceeds at tree-level via the $W^{ \pm}$exchange. The formula below describes this contribution in the Fermi approximation, with leading order corrections to the $W$ propagator, and dominant QED one-loop contributions,

$$
\begin{aligned}
&\left.\Gamma^{l}\right|_{S M}=\Gamma_{\text {tree }}^{W^{ \pm}}= \frac{G_{F}^{2} m_{\tau}^{5}}{192 \pi^{3}} f\left(\frac{m_{l}^{2}}{m_{\tau}^{2}}\right)\left(1+\frac{3 m_{\tau}^{2}}{5 m_{W}^{2}}-2 \frac{m_{l}^{2}}{m_{W}^{2}}\right) \times \\
&\left(1+\frac{\alpha\left(m_{\tau}\right)}{2 \pi}\left(\frac{25}{4}-\pi^{2}\right)\right), \\
& f(x)=1-8 x+8 x^{3}-x^{4}-12 x^{2} \ln x .
\end{aligned}
$$

We will denote the SM contribution in short as $\Gamma_{0}^{l}$, skipping here and below the superscript $l$ if not necessary.
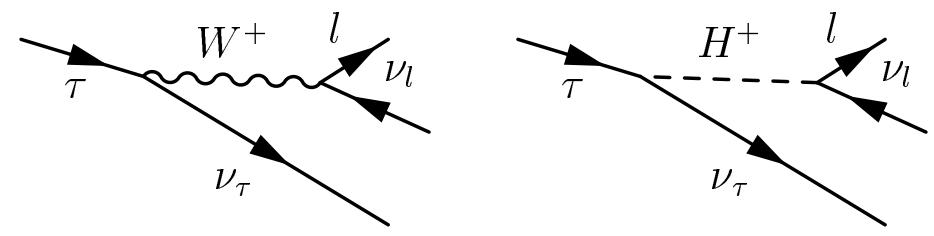

Figure 3: Tree-level contributions to the $\tau$ leptonic decays. The $W^{ \pm}$exchange in the $S M$ (on the left) and the $H^{ \pm}$exchange in $2 H D M$ (on the right).

In $2 \mathrm{HDM}$ there is, in addition, a tree contribution due to the exchange of the charged Higgs boson, Fig. 3 (right). This new contribution is given by

$$
\Gamma_{\text {tree }}^{H^{ \pm}}=\Gamma_{0}\left[\frac{m_{\tau}^{2} m_{l}^{2} \tan ^{4} \beta}{4 M_{H^{ \pm}}^{4}}-2 \frac{m_{l} m_{\tau} \tan ^{2} \beta}{M_{H^{ \pm}}^{2}} \frac{m_{l}}{m_{\tau}} \kappa\left(\frac{m_{l}^{2}}{m_{\tau}^{2}}\right)\right],
$$

where

$$
\kappa(x)=\frac{g(x)}{f(x)}, \quad g(x)=1+9 x-9 x^{2}-x^{3}+6 x(1+x) \ln (x) .
$$

Note that the second term is coming from the interference with the SM amplitude and it is much more important than the first one, that is suppressed by a factor $m_{\tau}^{2} \tan ^{2} \beta / 8 M_{H^{ \pm}}^{2}$. Note that such suppression can be compensated by a very large $\tan \beta$ only.

In $2 \mathrm{HDM}$ there are also one-loop contributions involving neutral as well as charged Higgs and Goldstone bosons. All these contributions are included in the $G_{F}$ scheme as follows: 


$$
\begin{aligned}
\Gamma_{1}^{l}= & \Gamma_{0}^{l}\left(1+\delta Z_{L \tau}+\delta Z_{L l}+\delta Z_{L \nu_{\tau}}+\delta Z_{L \nu_{l}}\right)+\Gamma_{\text {loops }}^{W^{ \pm}} \\
& +\Gamma_{\text {tree }}^{H^{ \pm}}+\Gamma_{\text {loops }}^{H^{ \pm}}+\Gamma_{C T}^{H^{ \pm}},
\end{aligned}
$$

where the first term corresponds to the SM prediction, $Z_{L f}=1+\delta Z_{L f}$ are the renormalisation constants for the left component of the fermion $f$ and $\Gamma_{\text {loops }}^{W^{ \pm}}$corresponds to the one-loop corrections to the $W^{ \pm}$exchange tree-level amplitude. The $H^{ \pm}$exchange tree-level contribution and its one-loop and counterterm corrections are described by $\Gamma_{\text {tree }}^{H^{ \pm}}, \Gamma_{\text {loops }}^{H^{ \pm}}$and $\Gamma_{C T}^{H^{ \pm}}$, respectively.

The tree-level $H^{ \pm}$contribution is numerically small and the radiative corrections to this amplitude will be neglected here. Taking this into account we will just consider the tree-level contribution eq.(13), implying that

$$
\Gamma_{\text {loops }}^{H^{ \pm}}=\Gamma_{C T}^{H^{ \pm}}=0 .
$$

\section{One-loop 2HDM(II) corrections}

We evaluate, in the 't Hooft-Feynman's gauge, the one-loop contributions coming from the $2 \mathrm{HDM}(\mathrm{II})$ to the quantities $\Delta^{l}$, using definitions and conventions for one-loop integrals of [21]. We will take into account the fact that the $H^{ \pm}$and $W^{ \pm}$masses are very large compared with the leptonic masses and external momenta, and we will neglect masses of muon and electron in the loop calculation. This means that the obtained one-loop corrections are universal, i.e. they do not depend whether decay into $e$ or $\mu$ is considered, so $\Delta_{\text {oneloop }}^{\mu}=\Delta_{\text {oneloop }}^{e}=\Delta_{\text {oneloop. }}$. Moreover, we will focus on large $\tan \beta$ enhanced contributions.

\section{$5.1 \quad$ Renormalisation constants}

In order to evaluate the $2 \mathrm{HDM}$ contributions to the fermion fields renormalisation constants, one has to compute the self-energies coming from the diagrams shown in Fig. 4 ,
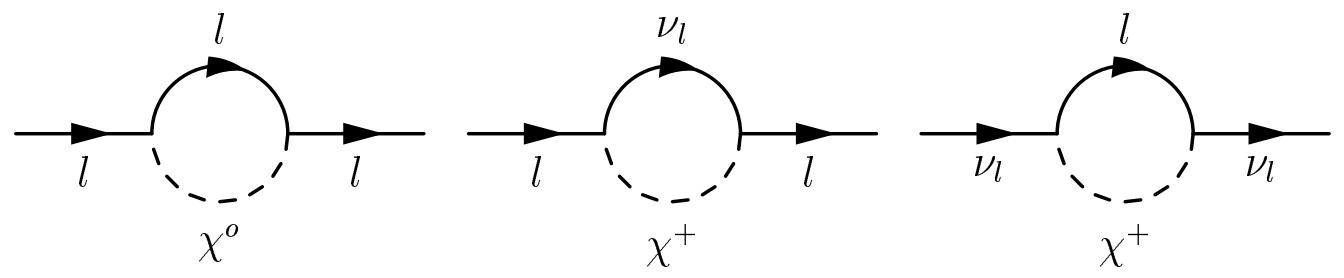

Figure 4: Two-point diagrams contributing to the fermion fields renormalisation. Here $\chi^{o}=h, H, A, G^{o}$ and $\chi^{+}=H^{+}, G^{+}$. 
Charged lepton self-energies. There are two kinds of contributions, one involving the exchange of a neutral boson and a second one involving a charged boson. The latter ones are numerically negligible since they are proportional to $m_{l}^{2} / M_{W}^{2}$ and $m_{l}^{2} / M_{H^{ \pm}}^{2}$ (for $\chi^{+}=G^{+}$and $H^{+}$, respectively).

Therefore we will consider the corrections coming from neutral Higgs and Goldstone bosons only. Since these corrections are proportional to $m_{l}^{2}$ we will take into account just the contributions to the self-energy of $\tau$. We obtain

$$
\begin{aligned}
\delta Z_{L e} & =\delta Z_{L \mu}=0 \\
\delta Z_{L \tau} & =\Delta_{\tau}^{h}+\Delta_{\tau}^{H}+\Delta_{\tau}^{A}+\Delta_{\tau}^{G^{o}} \\
\Delta_{\tau}^{h} & =-\frac{G_{F} m_{\tau}^{2}}{8 \sqrt{2} \pi^{2}} \frac{\sin ^{2} \alpha}{\cos ^{2} \beta} \mathcal{B}\left(m_{\tau}^{2} ; M_{h}^{2}, m_{\tau}^{2}\right) \\
\Delta_{\tau}^{H} & =-\frac{G_{F} m_{\tau}^{2}}{8 \sqrt{2} \pi^{2}} \frac{\cos ^{2} \alpha}{\cos ^{2} \beta} \mathcal{B}\left(m_{\tau}^{2} ; M_{H}^{2}, m_{\tau}^{2}\right) \\
\Delta_{\tau}^{A} & =-\frac{G_{F} m_{\tau}^{2}}{8 \sqrt{2} \pi^{2}} \tan ^{2} \beta \mathcal{B}\left(m_{\tau}^{2} ; M_{A}^{2}, m_{\tau}^{2}\right) \\
\Delta_{\tau}^{G^{o}} & =-\frac{G_{F} m_{\tau}^{2}}{8 \sqrt{2} \pi^{2}} \mathcal{B}\left(m_{\tau}^{2} ; M_{Z}^{2}, m_{\tau}^{2}\right) \simeq 0,
\end{aligned}
$$

where we use the following abbreviation

$$
\mathcal{B}()=\left[B_{0}+B_{1}+4 m_{\tau}^{2} B_{0}^{\prime}+2 m_{\tau}^{2} B_{1}^{\prime}\right]() .
$$

The $G^{o}$ contribution will be neglected since it is not $\tan ^{2} \beta$ enhanced.

Neutrino self-energies. In this case only the $H^{+}$and $G^{+}$contributions are involved and, since again these corrections are proportional to the mass of the lepton in the loop, we will just consider the corrections to the tauonic neutrino field renormalisation. We obtain

$$
\begin{aligned}
\delta Z_{L \nu_{e}} & =\delta Z_{L \nu_{\mu}}=0 \\
\delta Z_{L \nu_{\tau}} & =\Delta_{\nu_{\tau}}^{H^{+}}+\Delta_{\nu_{\tau}}^{G^{+}} \\
\Delta_{\nu}^{H^{+}} & =-\frac{G_{F} m_{\tau}^{2}}{4 \sqrt{2} \pi^{2}} \tan ^{2} \beta\left[B_{0}+B_{1}\right]\left(0 ; M_{H^{ \pm}}^{2}, m_{\tau}^{2}\right) \\
\Delta_{\nu}^{G^{+}} & =\frac{G_{F} m_{\tau}^{2}}{4 \sqrt{2} \pi^{2}}\left[B_{0}+B_{1}\right]\left(0 ; M_{W}^{2}, m_{\tau}^{2}\right) \simeq 0 .
\end{aligned}
$$

\subsection{One-loop three-point contribution}

The one-loop three-point diagrams contributing to $\Delta$ in the $2 \mathrm{HDM}(\mathrm{II})$ are presented in Fig. 5. We use here the following notation: $\chi^{0}=h, H, A, G^{o}$, $\chi^{+}=H^{+}, G^{+}$and $(V, \phi)=\left(G^{+}, Z\right),\left(W^{+}, h\right),\left(W^{+}, H\right) /\left(Z, G^{+}\right)$ 


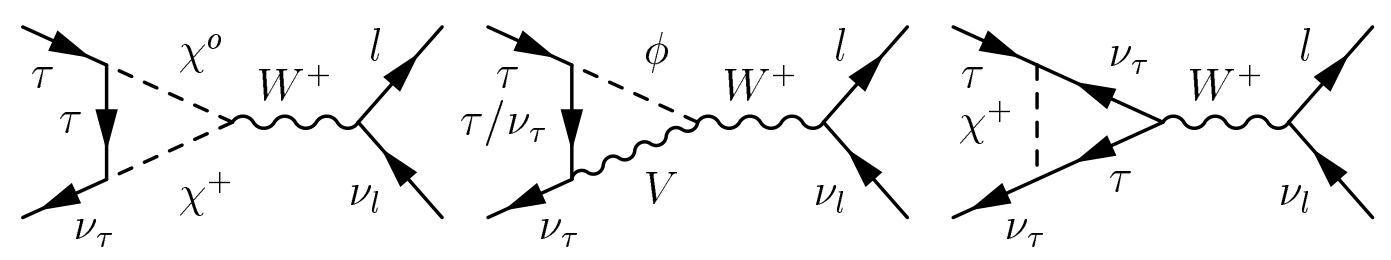

Figure 5: Three-point diagrams contributing to the $W^{ \pm} \tau \nu_{\tau}$ vertex correction. Similar diagrams exist for the $W^{ \pm} l \nu_{l}$ vertex. $\chi^{0}=h, H, A, G^{o}, \chi^{+}=H^{+}, G^{+}$ and $(V, \phi)=\left(G^{+}, Z\right),\left(W^{+}, h\right),\left(W^{+}, h\right) /\left(Z, G^{+}\right)$.

These $W^{ \pm} l \nu_{l}$ vertex corrections are proportional to the lepton mass and therefore we will consider only the radiative contributions to the $W^{ \pm} \tau \nu_{\tau}$ vertex. The different contributions coming from each diagram are as follows.

$\chi^{+}-\chi^{0}-\tau$ Loops. We have computed them (Fig. 1 left) in the limit of large $M_{H^{ \pm}}$and $M_{W}$. That means, we have obtained the complete expressions and keep only such terms that do not decouple in the limit $M_{H^{ \pm}}, M_{W} \gg m_{\tau}$. The resulting expressions are:

$$
\begin{aligned}
\Delta_{\text {loops }}^{H^{+} h} & =\frac{G_{F} m_{\tau}^{2}}{2 \sqrt{2} \pi^{2}} \tan \beta \frac{\sin \alpha}{\cos \beta} \cos (\alpha-\beta) C_{20}\left(m_{\tau}^{2}, m_{\nu_{\tau}}^{2} ; M_{h}^{2}, m_{\tau}^{2}, M_{H^{ \pm}}^{2}\right)+\ldots \\
\Delta_{\text {loops }}^{H^{+} A} & =\frac{G_{F} m_{\tau}^{2}}{2 \sqrt{2} \pi^{2}} \tan ^{2} \beta C_{20}\left(m_{\tau}^{2}, m_{\nu_{\tau}}^{2} ; M_{A}^{2}, m_{\tau}^{2}, M_{H^{ \pm}}^{2}\right)+\ldots \\
\Delta_{\text {loops }}^{H^{+} H} & =-\frac{G_{F} m_{\tau}^{2}}{2 \sqrt{2} \pi^{2}} \tan \beta \frac{\cos \alpha}{\cos \beta} \sin (\alpha-\beta) C_{20}\left(m_{\tau}^{2}, m_{\nu_{\tau}}^{2} ; M_{H}^{2}, m_{\tau}^{2}, M_{H^{ \pm}}^{2}\right)+\ldots \\
\Delta_{\text {loops }}^{G^{+} h} & =-\frac{G_{F} m_{\tau}^{2}}{2 \sqrt{2} \pi^{2}} \frac{\sin \alpha}{\cos \beta} \sin (\alpha-\beta) C_{20}\left(m_{\tau}^{2}, m_{\nu_{\tau}}^{2} ; M_{h}^{2}, m_{\tau}^{2}, M_{W}^{2}\right)+\ldots \simeq 0 \\
\Delta_{\text {loops }}^{G^{+} H} & =-\frac{G_{F} m_{\tau}^{2}}{2 \sqrt{2} \pi^{2}} \frac{\cos \alpha}{\cos \beta} \cos (\alpha-\beta) C_{20}\left(m_{\tau}^{2}, m_{\nu_{\tau}}^{2} ; M_{H}^{2}, m_{\tau}^{2}, M_{W}^{2}\right)+\ldots \simeq 0 \\
\Delta_{\text {loops }}^{G^{+} G^{o}} & =-\frac{G_{F} m_{\tau}^{2}}{2 \sqrt{2} \pi^{2}} C_{20}\left(m_{\tau}^{2}, m_{\nu_{\tau}}^{2} ; M_{Z}^{2}, m_{\tau}^{2}, M_{W}^{2}\right)+\ldots \simeq 0
\end{aligned}
$$

The three last contributions can be neglected in the large $\tan \beta$ limit.

$\boldsymbol{V}-\boldsymbol{\phi}-\boldsymbol{l}$ Loops. These contributions (Fig. 5 middle) are numerically negligible as do not contain any $\tan ^{2} \beta$ factor. Therefore, in our work

$$
\Delta_{\text {loops }}^{V \phi} \simeq 0 .
$$



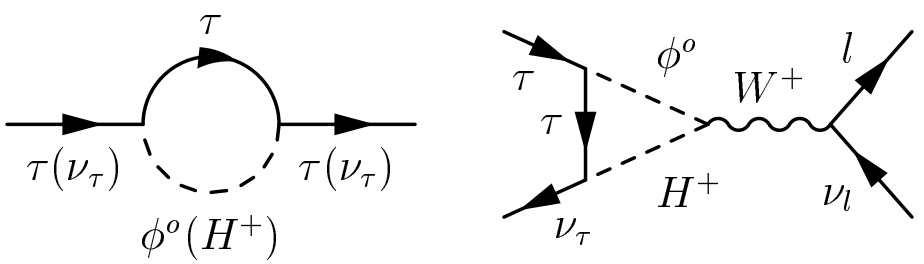

Figure 6: Dominant one-loop diagrams contributing to leptonic $\tau$ decays in the limit of large $\tan \beta$, here $\phi^{\circ}=h, H, A$.

$\boldsymbol{\tau}-\boldsymbol{\nu}_{\boldsymbol{\tau}}-\boldsymbol{\chi}^{+}$Loops. We have computed these contributions (Fig. [5 right) and checked that they decouple in the limit of very heavy charged Higgs boson and $W^{ \pm}$boson, as their leading terms in this limit are proportional to $\frac{m_{\tau}^{2}}{M_{H^{ \pm}}^{2}}$ or $\frac{m_{\tau}^{2}}{M_{W}^{2}}$. Therefore

$$
\Delta_{\text {loops }}^{\nu_{l} \chi^{+}} \simeq 0
$$

\subsection{One-loop box diagrams}

The one-loop box diagrams also contribute to the $\tau$ leptonic decays. All of these diagrams involve the exchange of a charged Higgs boson or a $W^{ \pm}$ boson. They can be safely neglected due to the mass dimension of the $D$ integrals that describe these diagrams, namely

$$
D_{0} \simeq \frac{1}{M^{4}}, D_{\mu} \simeq \frac{1}{M^{3}}, D_{\mu \nu} \simeq \frac{1}{M^{2}}, D_{\mu \nu \rho} \simeq \frac{1}{M}, D_{\mu \nu \rho \gamma} \simeq \mathcal{O}\left(M^{0}\right) .
$$

Since $M_{H^{ \pm}}$and $M_{W}$ are very large as compared to $m_{\tau}$, they will drive the mass dependence of the integrals, so $M=M_{H^{ \pm}}, M_{W}$. Therefore only the terms proportional to $D_{\mu \nu \rho \gamma}$ do not decouple and give relevant contributions. However, in the considered case of $\tau$ decays there are no such contributions. Therefore we can neglect box diagrams altogether:

$$
\Delta_{\text {loops }}^{\text {box }} \simeq 0
$$

\subsection{Final expression for one-loop contribution}

Taking all this into account, the dominant diagrams in the limit of large $\tan \beta$ are reduced to the ones drawn in Fig. 6. The contributions coming from these diagrams are

$$
\Delta_{\text {oneloop }}=\frac{G_{F} m_{\tau}^{2}}{8 \sqrt{2} \pi^{2}} \tan ^{2} \beta \times
$$




$$
\begin{aligned}
& {\left[-\cos ^{2}(\beta-\alpha) \mathcal{B}\left(m_{\tau}^{2} ; M_{h}^{2}, m_{\tau}^{2}\right)\right.} \\
& -\mathcal{B}\left(m_{\tau}^{2} ; M_{A}^{2}, m_{\tau}^{2}\right) \\
& -\sin ^{2}(\beta-\alpha) \mathcal{B}\left(m_{\tau}^{2} ; M_{H}^{2}, m_{\tau}^{2}\right) \\
& -2\left[B_{0}+B_{1}\right]\left(0 ; M_{H^{ \pm}}^{2}, m_{\tau}^{2}\right) \\
& +4 \cos ^{2}(\beta-\alpha) C_{20}\left(m_{\tau}^{2}, m_{\nu_{\tau}}^{2} ; M_{h}^{2}, m_{\tau}^{2}, M_{H^{ \pm}}^{2}\right) \\
& +4 C_{20}\left(m_{\tau}^{2}, m_{\nu_{\tau}}^{2} ; M_{A}^{2}, m_{\tau}^{2}, M_{H^{ \pm}}^{2}\right) \\
& \left.+4 \sin ^{2}(\beta-\alpha) C_{20}\left(m_{\tau}^{2}, m_{\nu_{\tau}}^{2} ; M_{H}^{2}, m_{\tau}^{2}, M_{H^{ \pm}}^{2}\right)\right] .
\end{aligned}
$$

An easy to handle expression can be obtained from eq.(24) for neutral Higgs masses larger that the $\tau$ mass, $M_{\phi^{\circ}} \geq m_{\tau}$. Notice that no assumption on the Higgs spectrum is made ${ }^{5}$. In this limit, we get ${ }^{6}$

$$
\begin{aligned}
\Delta_{\text {oneloop }} \approx \frac{G_{F} m_{\tau}^{2}}{8 \sqrt{2} \pi^{2}} \quad \tan ^{2} \beta & \tilde{\Delta} \\
\tilde{\Delta}=\quad & {\left[-\left(\ln \left(\frac{M_{H^{+}}^{2}}{m_{\tau}^{2}}\right)+F\left(R_{H^{ \pm}}\right)\right)\right.} \\
+ & \frac{1}{2}\left(\ln \left(\frac{M_{A}^{2}}{m_{\tau}^{2}}\right)+F\left(R_{A}\right)\right) \\
+ & \frac{1}{2} \cos ^{2}(\beta-\alpha)\left(\ln \left(\frac{M_{h}^{2}}{m_{\tau}^{2}}\right)+F\left(R_{h}\right)\right) \\
& \left.+\frac{1}{2} \sin ^{2}(\beta-\alpha)\left(\ln \left(\frac{M_{H}^{2}}{m_{\tau}^{2}}\right)+F\left(R_{H}\right)\right)\right]
\end{aligned}
$$

where $R_{\phi} \equiv M_{\phi} / M_{H^{ \pm}}$and

$$
F(R)=-1+2 \frac{R^{2} \ln R^{2}}{1-R^{2}}
$$

Some useful limits of the $F$ function are:

$$
F(R \ll 1) \sim-1, F(R=1)=-3, F(R \gg 1) \sim-\left(1+2 \ln R^{2}\right) .
$$

The above expression depends logarithmically on the ratios of the mass of each Higgs boson to the mass of tau lepton. However, this may be misleading as in fact there is no dependence on mass of the tau lepton in the above expression. Indeed, one can see this by looking at other useful form of $\tilde{\Delta},{ }^{7}$

$$
\tilde{\Delta}=3+\frac{1}{2}\left(G\left(R_{A}\right)+\cos ^{2}(\beta-\alpha) G\left(R_{h}\right)+\sin ^{2}(\beta-\alpha) G\left(R_{H}\right)\right),
$$

\footnotetext{
${ }^{5}$ We generalised here the result in [9] where assumptions were made $M_{H^{ \pm}}, M_{A} \gg M_{h}$ and $\alpha=\beta$.

${ }^{6}$ In agreement with [22] result derived in the context of the MSSM.

7 We thank M. Misiak for this suggestion.
} 
where

$$
G(R)=\ln R^{2}+F(R) .
$$

In the following, to explore the phenomenological consequences of the large $\tan \beta$ enhanced $2 \mathrm{HDM}(\mathrm{II})$ radiative corrections to the leptonic $\tau$ decays, we will use both the exact and approximated expressions (24) and (25] 28), respectively.

\subsection{One-loop corrections for some interesting scenarios}

In some phenomenologically interesting scenarios, the expressions (2528) can be simplified. In the case of light $h$ and $\sin ^{2}(\beta-\alpha)=0, \tilde{\Delta}$ does not depend on $M_{H}$ and two limits are worth to be studied,

$M_{A}=M_{H^{ \pm}} \rightarrow \tilde{\Delta}=\ln \frac{M_{h}}{M_{H^{ \pm}}}+1$ and $M_{A} \ll M_{H^{ \pm}} \rightarrow \tilde{\Delta}=\ln \frac{M_{h}}{M_{H^{ \pm}}}+\ln \frac{M_{A}}{M_{H^{ \pm}}}+2$.

Notice, that when $h$ does not couple to gauge bosons and therefore the Higgsstrahlung process at LEP is not sensitive to such Higgs boson, the leptonic tau decays have maximal sensitivity to $h$ as $\tilde{\Delta}$ depends logarithmically on its mass, without any suppression factor.

If $A$ is light and $\sin ^{2}(\beta-\alpha)=1$, one obtains the same expression for $\tilde{\Delta}$ that in the previous case with obvious replacing $h \rightarrow A$ and $A \rightarrow H$. Therefore any analysis with $h$ light and $\sin ^{2}(\beta-\alpha)=0$ can be easily translated to the case of light $A$ and $\sin ^{2}(\beta-\alpha)=1$.

The useful expression which holds for arbitrary $\sin (\beta-\alpha)$ and degenerate $H, A, H^{ \pm}$(with a common mass $M$ ) is:

$$
\tilde{\Delta}=\cos ^{2}(\beta-\alpha)\left[\ln \frac{M_{h}}{M}+1\right] .
$$

We see that in a SM-like scenario, with light $h, \sin ^{2}(\beta-\alpha)=1$ and very heavy degenerate additional Higgs bosons, $\widetilde{\Delta}$ goes to zero, what signals a clear decoupling.

\section{$6 \quad$ Numerical analysis}

In this section we analyse the dependence of the $2 \mathrm{HDM}(\mathrm{II})$ one-loop corrections obtained in the previous sections for the leptonic $\tau$ decays on the different Higgs bosons masses and mixing angles. First we stress that typically the one-loop contribution dominates the $2 \mathrm{HDM}(\mathrm{II})$ effects. They are, for fixed value of large $\tan \beta$ and in the interesting region of parameter space, five orders of magnitude larger than the corresponding tree-level $\mathrm{H}^{ \pm}$contribution to $\Delta^{e}$, and one or two orders of magnitude larger for the $\Delta^{\mu}$. Therefore, although we will include all contributions in the numerical analysis, the 
main features of the 2HDM effects are described by the one-loop correction in eq.(25). In the following only results for muon decay channel will be presented. We stress however that the obtained one-loop corrections are the same for the electron and muon channels.

In eq. (25) one can distinguish two contributions, one coming from the charged Higgs alone and the other one involving also the neutral Higgs bosons. The former is always negative and it becomes more negative for a larger charged Higgs mass. The latter is typically positive and it grows with the neutral Higgs masses. In this way the total 2HDM(II) one-loop effects, being a sum of two contributions of the same order and with different signs, will be large when one of these contributions dominates. Since the modulus of both corrections grow with the Higgs masses one expects large one-loop effects in two cases: (i) heavy $H^{ \pm}$and light $\phi^{\circ}$ (large negative corrections) and (ii) light $H^{ \pm}$and heavy $\phi^{o}$ (large positive corrections). Taking into account the lower bound for $M_{H^{ \pm}}$coming from $b \rightarrow s \gamma, M_{H}^{ \pm}$above 490 $\mathrm{GeV}$, one expects to get large radiative effects in case (i) only. Note that in this (i) case the $\tilde{\Delta}$ (loop contribution) is negative, as well as the tree-level $H^{ \pm}$exchange, eq.(13).

We will focus on two scenarios of a special phenomenological interest: with a light scalar $h$ and with a light pseudoscalar $A$. As all contributions considered here are proportional to $\tan ^{2} \beta$, they will be plotted for $\tan \beta=1$, to be rescaled by $\tan ^{2} \beta$.

\subsection{Light scalar Higgs boson, $h$}

First we will consider a scenario with a light scalar boson, $h$, with mass $M_{h}$ below $114 \mathrm{GeV}$, and degenerate heavy Higgs bosons, with masses $M_{A}=$ $M_{H}=M_{H^{+}}=M$, above $300 \mathrm{GeV}$. For such a light Higgs boson $h$, its couplings to gauge bosons are constrained by LEP data as shown in Fig. 1] (left), lying between 0 and $\left.\sin ^{2}(\beta-\alpha)\right|_{\max }$. Note that for arbitrary $\sin (\beta-\alpha)$ and degenerate $H, A, H^{ \pm}$the equation (31) holds.

In the light $h$ scenario instead of the degenerated heavy additional Higgs bosons, one can also consider a spectrum with SM-like Higgs boson $H$ ( i.e. with couplings to the gauge bosons as for the SM Higgs, namely $\chi_{V}^{H}=1$ or $\sin (\beta-\alpha)=0)$. It is reasonable to assume that such Higgs boson has a mass in the region expected for the SM Higgs boson, say $M_{H}=115 \mathrm{GeV}$, although as follows from eq. (25) nothing depends on this mass. On contrary, one gets here a clear dependence on mass of $h$ :

$$
\tilde{\Delta}=\ln \frac{M_{h}}{M}+1
$$

The different contributions to $\Delta \propto \tilde{\Delta} \tan ^{2} \beta$ are plotted in Fig. 7 (left) for $M_{h}=5$ and $70 \mathrm{GeV}$, for degenerate heavy Higgs bosons. The total ( i.e.sum of the tree and one-loop) contributions are plotted using solid lines, while the one-loop contributions using dashed lines, respectively. As 
can be seen, the $H^{ \pm}$tree-level effect is important for low $M$ but the oneloop contribution becomes dominant for $M \geq 500 \mathrm{GeV}$. In particular, the logarithmic dependence on $M$ coming from the one-loop corrections is clearly seen. Notice that curves are plotted for $\sin ^{2}(\beta-\alpha)=0$ and $\left.\sin ^{2}(\beta-\alpha)\right|_{\text {max }}$, the maximum value allowed by LEP data for a given $M_{h}$ value. For $h$ mass equal to $5 \mathrm{GeV}$ the results for different $\sin ^{2}(\beta-\alpha)$, laying between 0 and 0.02 , can not be distinguished.

\section{Contribution to $\mathrm{Br} \_\mathrm{mu}$ in $2 \mathrm{HDM}(\mathrm{II})$}

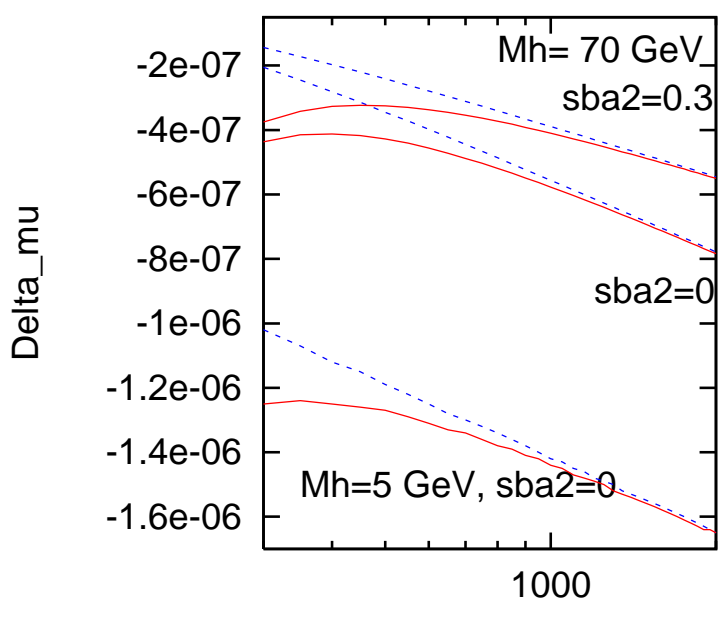

$\mathrm{M}, \mathrm{GeV}$

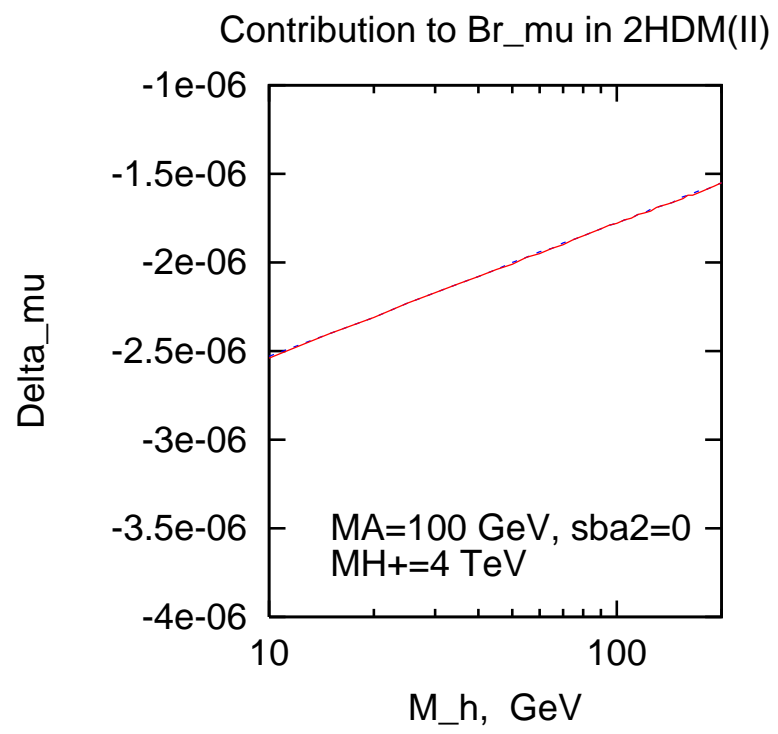

Figure 7: The total (solid line) and one-loop (dashed line) contributions to $\Delta$ for $\tan \beta=1$. Left: Results for $M_{h}=5$ and 70 GeV are plotted as a function of $M=M_{A}=M_{H}=M_{H^{ \pm}}$. Results for mass of $70 \mathrm{GeV}$ for $\sin ^{2}(\beta-\alpha)=0$ and 0.3 are presented by the bottom and upper lines, respectively. Right: Results for $M_{A}=100 \mathrm{GeV}, M_{H^{ \pm}}=4 \mathrm{TeV}$ and $\sin ^{2}(\beta-\alpha)=0\left(M_{H}\right.$ is arbitrary), as a function of $M_{h}$ are shown.

The dependence of $\Delta$ on the light Higgs mass can be seen in Fig. 7 (left) by comparing the results obtained for $M_{h}=5$ and $70 \mathrm{GeV}$. This dependence is explicitly presented in Fig. 7 (right) where the contributions are plotted as a function of $M_{h}$, for $M_{A}=100 \mathrm{GeV}, M_{H^{ \pm}}=4 \mathrm{TeV}, \sin (\beta-\alpha)=0$. The $2 \mathrm{HDM}(\mathrm{II})$ one-loop corrections decrease logarithmically with increasing $M_{h}$ as described by eq. (32). So the lighter $h$ the larger the one-loop corrections. One can see that $\Delta$ decreases linearly with increasing $\sin ^{2}(\beta-\alpha)$, in agreement with eq. (31).

In the case with the SM-like $H$ we have $\sin ^{2}(\beta-\alpha)=0$, then $\Delta$ becomes insensitive to the value of $M_{H}$, see eq.(25) and discussion above. Therefore all above results obtained for the $\sin ^{2}(\beta-\alpha)=0$ case hold also here for the SM-like $H$. 


\subsection{Light pseudoscalar, $A$}

In the case where the pseudoscalar, $A$, is light and the two neutral scalars are degenerate $M_{h}=M_{H}$, the $\tilde{\Delta}$ does not depend on $\sin ^{2}(\beta-\alpha)$. For $M_{h}=M_{H}=M_{H^{ \pm}}=M$ we get a simple formula

$$
\tilde{\Delta}=\ln \frac{M_{A}}{M}+1
$$

It is similar to the formula obtained for the discussed above case of light $h$ for $\sin ^{2}(\beta-\alpha)=0$, with obvious change $M_{h}$ to $M_{A}$. Therefore we will not present the results corresponding to such light $A$ case.

There is an interesting light $A$ scenario where in addition to $A$ also $h$ is not very heavy. We call this case a light $\mathrm{A} \& \mathrm{~h}$ scenario. Here we choose the $h$ mass to be equal to $100 \mathrm{GeV}$ to avoid a direct conflict with LEP data presented in Fig. 1 (right). In Fig. 8 the total contribution to $\Delta$ is plotted as solid lines, while one-loop corrections as dashed lines, respectively. Also in this light $\mathrm{A} \& \mathrm{~h}$ scenario we see that the one-loop effects dominate for large $M$ scale. The largest deviation from the SM prediction occurs for $\sin ^{2}(\beta-\alpha)=0$.

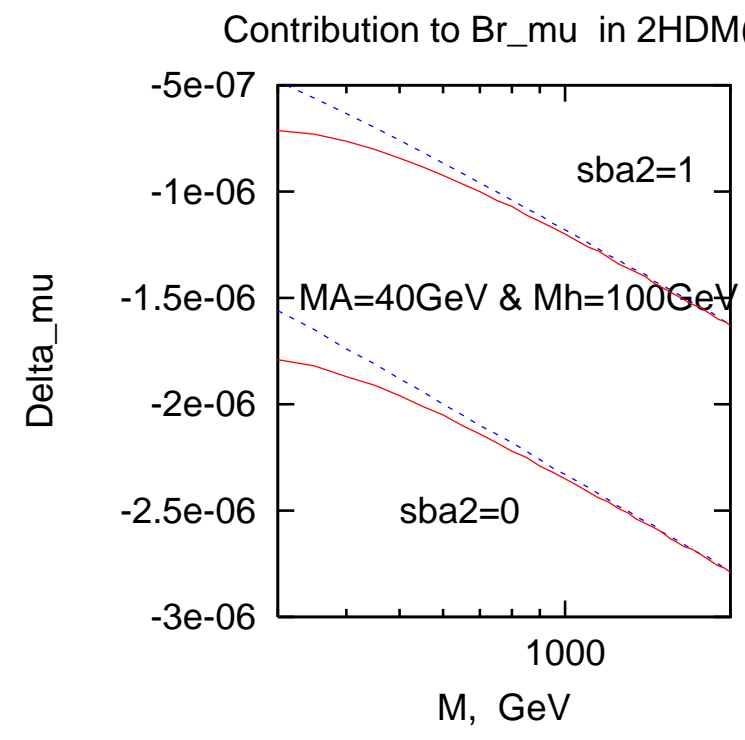

Figure 8: The total (solid line) and one-loop (dashed line) contributions to $\Delta$ for $\tan \beta=1$. Results for $M_{A}=40 \mathrm{GeV}$ and $M_{h}=100 \mathrm{GeV}$ are shown for $\sin ^{2}(\beta-\alpha)=0$ and 1 , bottom and upper lines, respectively.

\subsection{Comparison of the exact and approximated results}

Results based on eq.(24) and the approximation eq.(25) have been plotted together in all the figures, being clearly indistinguishable. Therefore, the simple approximated formulae eqs.(2528) can be used to describe the $2 \mathrm{HDM}$ (II) 
one-loop corrections to the $\tau$ leptonic decays in the whole considered range of parameters.

\section{Constraining $2 \mathrm{HDM}(\mathrm{II})$ by the $\tau$ decay data}

In this section we use the leptonic $\tau$ decay data to constrain 2HDM(II) parameters. The complementarity between LEP processes used for direct searches of light Higgs bosons and indirect measurements based on the leptonic $\tau$ decays will be exploited to explore the "pessimistic", for the direct searches, scenarios. In particular the case $\sin ^{2}(\beta-\alpha)=0$ will be studied, since in this scenario the Higgsstrahlung and VV fusion processes for $h$ are suppressed. The $95 \%$ CL bounds for $\Delta$ derived by us in Sect. 3 allow to set upper bounds on $\tan \beta$ (Yukawa couplings) for both the light $h$ or $A$ scenarios. We provide also exclusion for $\left(M_{h}, M_{A}\right)$ plane for various values of $\tan \beta$ and $\sin (\beta-\alpha)$.

In addition, we obtain from $\tau$ decays new lower and upper bounds on the charged Higgs boson mass as a function of $\tan \beta$.

\subsection{Constraints on the Yukawa couplings of the light- est neutral Higgs bosons}

The upper limits on $\tan \beta$ (Yukawa coupling $\chi_{d}$ ) for light $h$ and light $A$ scenarios are shown in Fig. 9] and 10, respectively.

In the "pessimistic" light $h$ scenario with $\sin ^{2}(\beta-\alpha)=0$, the leptonic $\tau$ decay data can be exploited to set upper limits on the Yukawa couplings as a function of $M_{h}$. They can be compared with limits coming from other experiments.

In Fig. 9] the upper limits on the $\tan \beta$ (Yukawa couplings) for light $h$, assuming $\sin (\beta-\alpha)=0$, derived from the leptonic $\tau$ decay data are presented. One can see that these data provide upper limits on $\tan \beta$ in region unaccessible by other experiments, namely for mass above $45 \mathrm{GeV}$.

As an opposite case to the light $h$ scenario, one can consider the case with a light pseudoscalar $A$. If $\sin (\beta-\alpha)=0, M_{A}$ can be low if $h$ is heavy enough to suppress the associated $(h, A)$ production. The Yukawa couplings of $A$ can be then constrained just by the Yukawa process with $f \bar{f} A$ final state and the Upsilon decay, $\Upsilon \rightarrow A \gamma$, for a very light Higgs boson $A$. Also in this case the leptonic $\tau$ decays can be used to set upper limits on the Yukawa coupling $(\tan \beta)$ as a function of $M_{A}$, see Fig. 10, The right panel shows the region around mass of $A$ equal $10 \mathrm{GeV}$.

Since this scenario can be relevant in explaining $(g-2)_{\mu}$ data, we plot in Fig. 11 the upper limits for $\tan \beta$ from the leptonic $\tau$ decay and the allowed region from the newest $g-2$ for muon data, and for a comparison all other existing upper limits for $A$. Degenerate masses of $h, H, H^{+8}$ were assumed

\footnotetext{
$8 \sin (\beta-\alpha)$ is then arbitrary
} 
Exclusion 95\%C.L. for $\mathrm{h}$ in $2 \mathrm{HDM}(\mathrm{II})$

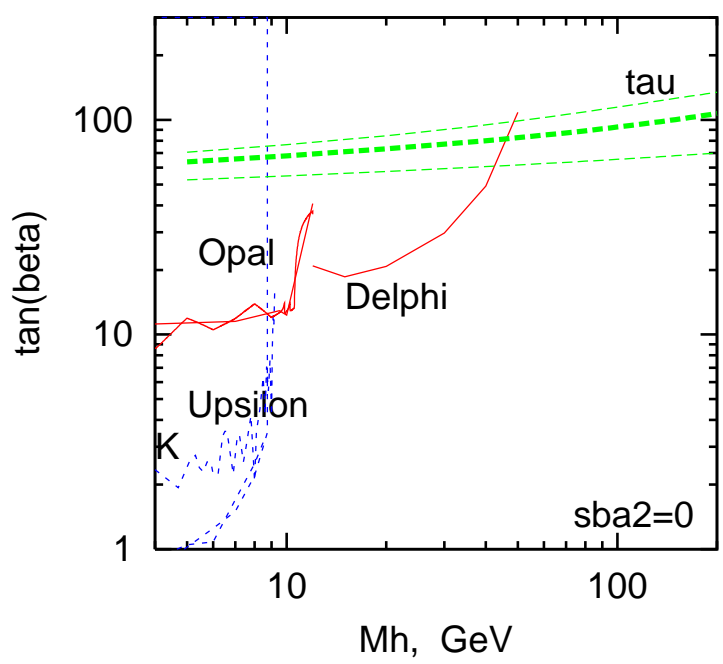

Figure 9: $95 \% C L$ upper limits from $\tau$ decay for $\tan \beta$ and $\sin (\beta-\alpha)=0$, as a function of $M_{h}$ compared to the existing upper limits from the Yukawa processes at LEP (Opal, Delphi) and the Upsilon decay. The two almost horizontal lines (in green) corresponds to $M_{A}=100 \mathrm{GeV}$, for $M_{H^{ \pm}}=500$ GeV and $4 \mathrm{TeV}$, upper and lower lines, respectively. The results for the degenerate $A$ and $H^{+}$with mass 4 TeV are plotted by using thicker line.

to be equal to 1 and $4 \mathrm{TeV}$, the corresponding upper limits are presented by the upper and lower lines, respectively.

\subsection{Constraints on a light $A \& h$ scenario}

A scenario with both $h$ and $A$ light is also of phenomenological interest. Since $\Delta$ can be large for low $M_{h}$ and $M_{A}$, the leptonic $\tau$ decay data can be used to constrain this scenario, in the $\left(M_{h}, M_{A}\right)$ parameter space. The comparison of these constraints with the ones coming from direct searches will reveal the importance of indirect ones from the leptonic $\tau$ decays.

In Fig. 12 the constrained regions in the $\left(M_{h}, M_{A}\right)$ plane, laying between axes and the corresponding curves, are shown for $\sin (\beta-\alpha)=0$ and $\tan \beta$ equal 60 and 90 . The excluded regions are symmetric in $M_{h}$ and $M_{A}$; they rule out the possibility of both $h$ and $A$ being very light. These constraints should be compared to the constraints shown in Fig. 1 (right). For large values of $M_{H^{ \pm}}$and $\tan \beta$ the $2 \mathrm{HDM}(\mathrm{II})$ one-loop effects can be very large and some of the regions of the parameter space allowed by direct searches can be excluded indirectly by using the leptonic $\tau$ decays. 
Exclusion 95\%C.L. for A in 2HDM(II)

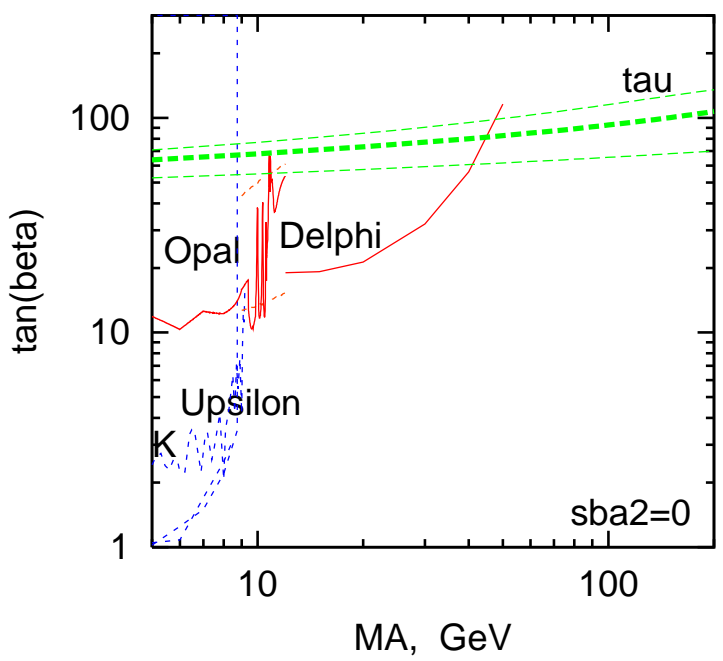

Exclusion 95\%C.L. for A in 2HDM(II)

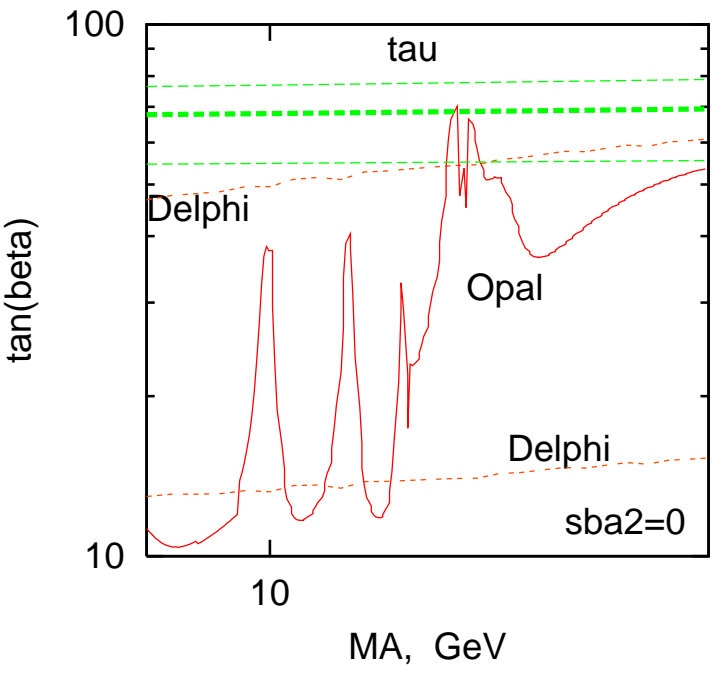

Figure 10: $95 \% C L$ upper limits from $\tau$ decay for $\tan \beta$ as a function of $M_{A}$ compared to the existing upper limits from Yukawa processes at LEP (OPAL, DELPHI) and Upsilon decay. The two almost horizontal lines (in green) corresponds to $M_{h}=100 \mathrm{GeV}$ and $M_{H^{ \pm}}=500 \mathrm{GeV}$ and $4 \mathrm{TeV}$, upper and lower lines, respectively. The results for degenerate $h$ and $H^{ \pm}$with mass 4 $\mathrm{TeV}$ are plotted in thicker line. Left: Mass range for A from 5 to $200 \mathrm{GeV}$, Right: Mass range for A from 8 to $12 \mathrm{GeV}$.

\subsection{Constraints on the charged Higgs-boson mass}

From the leptonic tau decays one can derive limits on the mass of charged Higgs boson as a function of $\tan \beta$. A standard derivation within the $2 \mathrm{HDM}$ (II) is based on the tree-level $\mathrm{H}^{+}$contribution for the leptonic tau decay into a muon. Such derivation can be found in almost all papers, both theoretical and experimental ones, devoted to this subject (see eg. [1]).

First, we apply such a standard method to derive from the tree-level contribution (for muon) only the lower mass limit for $\mathrm{H}^{+}$. By applying the obtained lowest value for the 95\% CL deviation from the SM prediction (eq. 1010) we updated the existing lower mass limit. We got the following limit

$$
M_{H^{ \pm}} \gtrsim 1.71 \tan \beta \mathrm{GeV}
$$

with coefficient 1.71 to be compared to the corresponding coefficients from [23] and [24], equal to 1.86 and 1.4, respectively. Note, that this is nothing else, up to the lepton mass ratio, what issued as the constraints on the Michel parameter $\eta$ in the 2HDM (II), see eg. $[1,25]$

Next, knowing that the one-loop corrections are typically more important than the tree contribution, we use them in the derivation of the mass limit for $H^{+}$. We observe that since $\Delta_{\text {oneloop }}$ grows with $M_{H^{ \pm}}$, this one-loop correction allows to put upper bounds on $M_{H^{ \pm}}$in scenarios with light neutral 


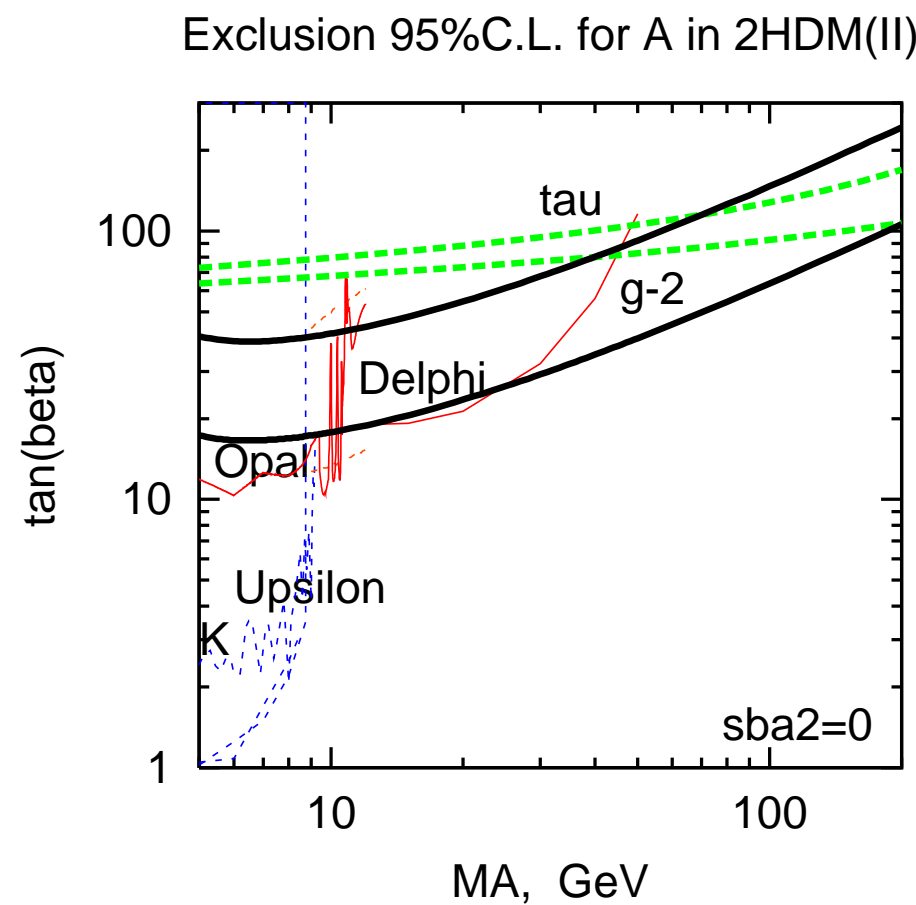

Figure 11: Upper limits for $\tan \beta$ from the leptonic $\tau$ decay (thick grey lines) and the allowed region from the newest $g-2$ for muon data (thick black lines), in comparison all other existing upper limits as a function of $M_{A}$. Degenerate masses of $h, H, H^{+}$were assumed to be equal to 1 and $4 \mathrm{TeV}$; the corresponding results from tau decay are given by the upper and lower thick grey lines, respectively.

Higgs bosons. In particular, for $\sin (\beta-\alpha)=0, \tilde{\Delta}$ goes as $\ln \left(M_{H^{ \pm}} / M_{h}\right)+$ $\ln \left(M_{H^{ \pm}} / M_{A}\right)$ (eq. 30) and therefore the lighter $h$ and $A$ the stronger upper bounds for $M_{H^{ \pm}}$.

In Fig. 13 (left) the individual lower and upper bounds on $M_{H^{ \pm}}$, as obtained from the tree-level $H^{ \pm}$exchange diagram only and from the one-loop contribution only, are plotted as a function of $\tan \beta$. The constraints based on the one-loop contributions are plotted for various masses of $h$, equal to 5, 20 and $100 \mathrm{GeV}$, assuming $\sin (\beta-\alpha)=0$ and a degeneracy in masses of $A$ and $H^{ \pm}$. Upper limits coming from the one-loop corrections are plotted both for the muon and electron decay channels, the limits from electron one is slightly weaker (dashed (green) lines). The relevant lower limits from the tree-level $H^{ \pm}$contribution is obtained only from the muon channel. The lower bound coming from $b \rightarrow s \gamma$ analysis is also shown for a comparison.

In Fig. 13 (right) we present results as described above for one particular mass of $h$ equal $20 \mathrm{GeV}$, together with a full bound based on a sum of tree and one-loop contribution (thick line). We see that a full bound gives both the lower and upper limits as a function of $\tan \beta$. In this figure we present 


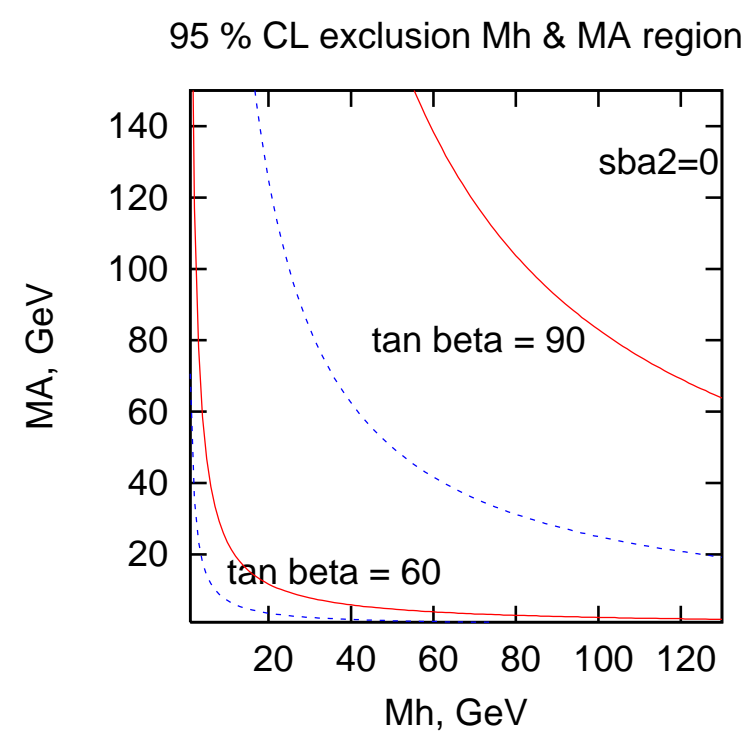

Figure 12: The excluded regions in the $\left(M_{h}, M_{A}\right)$ plane for $\sin ^{2}(\beta-\alpha)=0$. The excluded regions lay between axes and curves corresponding to $M_{H^{ \pm}}=$ $500 \mathrm{GeV}$ and $1 \mathrm{TeV}$, dashed and solid lines, and to $\tan \beta=60,90$, down and upper lines, respectively.

also results obtained for other mass of $A$, equal $100 \mathrm{GeV}$. The thin black dotted line corresponds to the limits obtained then, with all other 2HDM parameters as used above to obtain a thick line.

It is clear that the constraints for mass of $H^{+}$change drastically if the one-loop contributions are included in the analysis. In particular, the lower bound is higher than the tree-level limit, eq.(34). Only for the SM-like $h$ scenario, with $\sin (\beta-\alpha)=1$ and all other Higgs boson mass heavy and degenerate, the tree-level contribution gives a reliable estimation.

Based on results presented in Fig. 13 the restrictions can be set on $M_{H^{ \pm}}$ for large values of $\tan \beta(\tan \beta \geq 60)$. In particular, in a scenario with light $h$ and not so heavy $A, M_{H^{ \pm}}$should be lower than $3 \mathrm{TeV}$ for $\tan \beta=65$. Although large values of $\tan \beta$ are required, this upper bound to the charged Higgs mass is important due to the difficulty on setting upper bounds on masses of undiscovered particles.

\section{Summary and conclusions}

In this work we have computed the $2 \mathrm{HDM}(\mathrm{II})$ one-loop corrections to the leptonic $\tau$ decays. As a main result we have obtained that these one-loop effects are larger than the corresponding tree-level $H^{ \pm}$contribution in the relevant regions of the parameter space. Our analysis has been focused on the $\tan \beta$ enhanced contributions and an easy-to-handle formula has been 


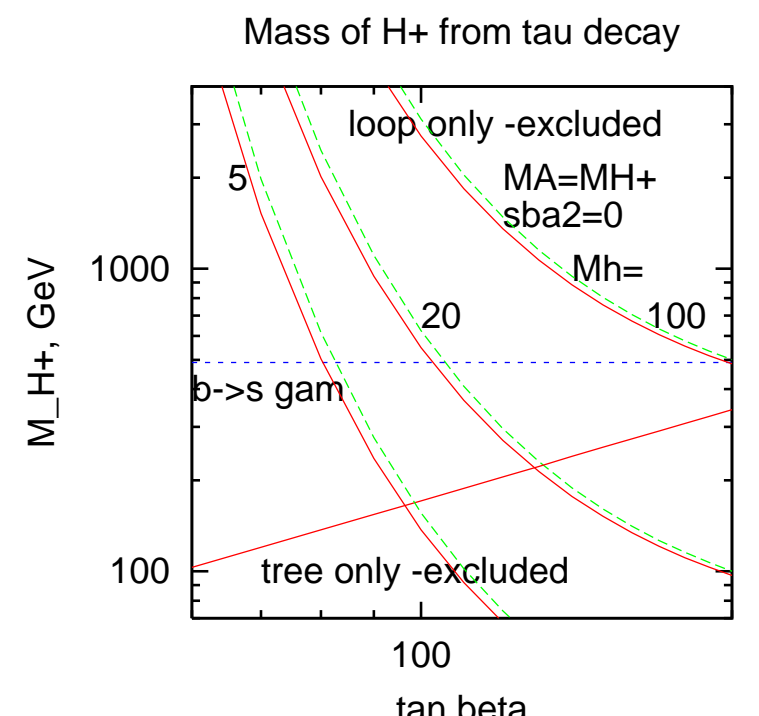

tan beta

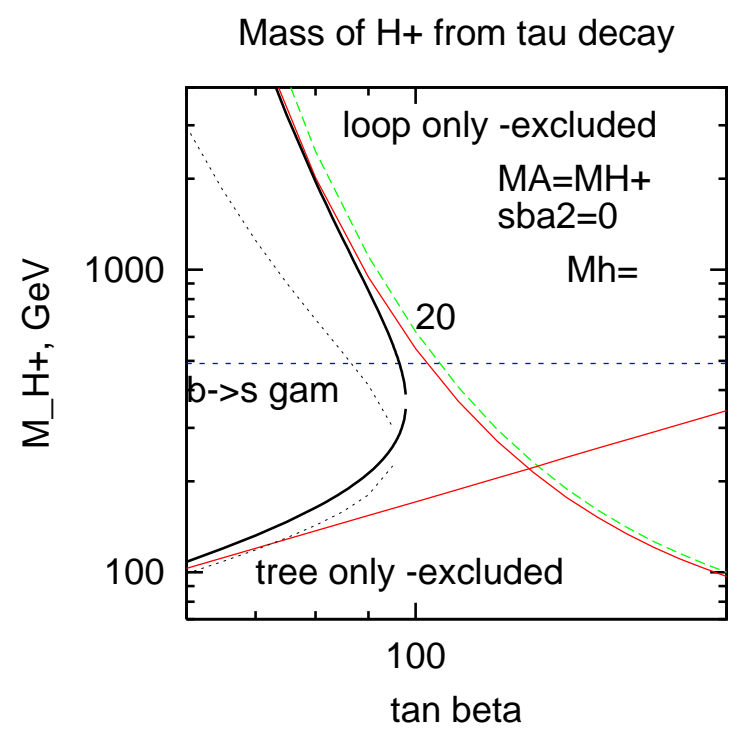

Figure 13: Limits on charged Higgs boson mass as a function of $\tan \beta$ obtained from leptonic tau decays. The upper limits from the one-loop contribution (dashed and solid lines correspond to the electron and muon channels) and lower limits (straight lines) from the tree $\mathrm{H}^{+}$exchange from muon channel are shown. Lower limit from $b \rightarrow s \gamma$ is also shown. Left: The upper limits obtained from the one-loop corrections only for $M_{h}=5,20,100 \mathrm{GeV}$ and $\sin ^{2}(\beta-\alpha)=0$, assuming $M_{A}=M_{H}^{+}$are presented. Right: The same as in Left for one mass $M_{h}=20 \mathrm{GeV}$, in addition in form of the full constraint from the total (loop plus tree) contribution, for degenerate masses $M_{A}=M_{H^{ \pm}}$ (thick solid line), and for $M_{A}=100 \mathrm{GeV}$ (thin dotted line) is presented.

obtained describing these one-loop effects in the approximation of the Higgs boson masses larger than the $\tau$ mass. This formula allows us to study all the 2HDM parameter space in a transparent way.

After the numerical analysis of the corrections, the constraints on the $2 \mathrm{HDM}(\mathrm{II})$ parameters from the leptonic $\tau$ decay data have been obtained in different scenarios. In particular the "pessimistic" scenarios for the direct searches of light $h$ Higgs bosons at LEP have been intensively analysed. From this analysis we have obtained upper limits on the Yukawa couplings for both light $h$ and light $A$ scenarios, constraining also the light $A \& h$ scenario. We have updated the existing in literature lower limits on $M_{H^{ \pm}}$, different from the ones coming from tree-level exchange only, and we have also obtained interesting upper limits on $M_{H^{ \pm}}$as a function of $\tan \beta$.

Therefore, one can conclude that leptonic $\tau$ decay data constrain $2 \mathrm{HDM}$ (II) scenarios with large $\tan \beta$, heavy $H^{ \pm}$and light neutral Higgs bosons.

Obviously, the large 2HDM(II) one-loop corrections found in this paper can have consequences for other type of processes, which will be analysed elsewhere. 


\section{Acknowledgement}

The authors thank Theory Group at CERN for a kind hospitality allowing us to work on a final version of the paper and M. J. Herrero for fruitful discussions. MK is grateful for important discussions with M. Misiak, Z. Wạs, B. Stugu and W. Marciano. This work was partially supported by the Polish Committee for Scientific Research,grant no. 1 P03B 04026 and project no. 115/E-343/SPB/DESY/P-03/DWM517/2003-2005 and the European Community's Human Potential Programme under contract HPRNCT-2000-00149 and and HPRN-CT-2002-00311 EURIDICE.

\section{References}

[1] S. Eidelman et al., Phys. Lett. B592, 1 (2004)

[2] J.F. Gunion, H.E. Haber, G.L. Kane and S. Dawson, The Higgs Hunter's Guide, Addison-Wesley Publishing Company, Reading, MA, 1990. J. F. Gunion and H. E. Haber, Phys. Rev. D 67 (2003) 075019 arXiv:hep-ph/0207010.

[3] The DELPHI Collaboration. DELPHI 2002-037-CONF-571.

G. Abbiendi et al. [OPAL Collaboration], Eur. Phys. J. C 23 (2002) 397 arXiv:hep-ex/0111010.

[4] P. Gambino and M. Misiak, Nucl. Phys. B 611, 338 (2001)

[5] The OPAL Collaboration. OPAL PHYSICS NOTE PN509 (ICHEP 04). D. Horvath [OPAL Collaboration], Nucl. Phys. A 721 (2003) 453.

[6] P. Chankowski, M. Krawczyk and J. Zochowski, Eur.Phys.J. C 11, 661 (1999).

[7] K. Cheung and O.C.W. Kong, Phys. Rev. D68 053003 (2003).

[8] J. Rosiek, Phys. Lett. B 252135 (1990)

[9] W. Hollik and T. Sack, Phys. Lett. B 284427 (1992)

[10] A. Akeroyd, A. Arhrib, E. M. Naimi, Phys.Lett. B490 119 (2000) and references therein.

[11] I. F. Ginzburg, M. Krawczyk and P. Osland, "Two-Higgs-doublet models with CP violation," arXiv:hep-ph/0211371.

I. F. Ginzburg and M. Krawczyk, "Symmetries of two Higgs doublet model and CP violation," arXiv:hep-ph/0408011.

[12] I. F. Ginzburg, M. Krawczyk and P. Osland, arXiv:hep-ph/0101331. Nucl. Instrum. Meth. A 472 (2001) 149 arXiv:hep-ph/0101229. 
[13] S. Kanemura, Y. Okada and E. Senaha, arXiv:hep-ph/0410048.

S. Kanemura, Y. Okada, E. Senaha and C. P. Yuan, arXiv:hep-ph/0408364.

S. Kanemura, S. Kiyoura, Y. Okada, E. Senaha and C. P. Yuan, Phys. Lett. B 558, 157 (2003) arXiv:hep-ph/0211308. arXiv:hep-ph/0209326.

[14] A. Arhrib, W. Hollik, S. Penaranda and M. Capdequi Peyranere, Phys. Lett. B 579, 361 (2004).

[15] G. W. Bennett et al. [Muon g-2 Collaboration], Phys. Rev. Lett. 92, 161802 (2004) arXiv:hep-ex/0401008,

H. Deng [Muon g-2 Collaboration], arXiv:hep-ex/0408148.

[16] A. Hocker, arXiv:hep-ph/0410081.

S. I. Eidelman, Acta Phys. Polon. B 34 (2003) 4571.

A. Nyffeler, Acta Phys. Polon. B 34, 5197 (2003).

[17] M. Krawczyk, "Precision muon g-2 results and light Higgs bosons in the 2HDM(II)," Acta Phys. Polon. B 33 (2002) 2621 arXiv:hep-ph/0208076.

[18] The OPAL Collaboration: G. Abbiendi, et al. Phys. Lett. B597 11 (2004).

[19] The OPAL Collaboration: G. Abbiendi, et al. CERN-PH-EP/2004-039 (hep-ex/0408097).

[20] M. Neubert, Renormalization-Group Improved Calculation of the $B \rightarrow$ $X_{s} \gamma$ Branching Ratio (hep-ph/0408179).

[21] W. Hollik, in Precision Tests of the Standard Electroweak Model, edited by P. Langacker (World Scientific, Singapore, 1995), p. 37-116.

[22] P.H. Chankowski, R. Hempfling and S. Pokorski, Phys.Lett. B333 403 (1994).

[23] M.T. Dova, J. Swain and L. Taylor, Phys. Rev. D58, 015005 (1998).

[24] A. Stahl and H. Voss, Z. Phys. C74, 73 (1997).

[25] A. Rouge, Eur. Phys. J. C 18 (2001) 491 arXiv:hep-ph/0010005. 